\title{
Improved Pareto/NBD Model and Its Applications in Customer Segmentation based on Personal Information Combination
}

\author{
Yanhong Guo, Hui Wang and Wei Liu \\ Faculty of Management and Economics, Dalian University of Technology, \\ Dalian, Liaoning, China, 116024 \\ guoyh@dlut.edu.cn,wanghui20081027@126.com,liuwei5712@163.com
}

\begin{abstract}
In recent years, the Pareto/NBD model has gotten the favor of many scholars because of its accurate ability of predicting the future customer purchase frequency. Firstly, the paper analyzed the disadvantages of Pareto/NBD model; Secondly, the paper put forward an improved Pareto/NBD model which integrated the customer personalized information in order to resolve the problem in the Pareto/NBD model and improve the accuracy of prediction; Thirdly, the paper used the real sales data of a Dalian Mall as the sample, empirically tested the superiority of the improved Pareto/NBD model to the original Pareto/NBD model. Finally, this paper combined with the improved Pareto/NBD model and the Gamma-Gamma model to predict each customer lifetime value, and put forward the segmentation method based on CLV and management strategy, help the enterprises implement differentiated marketing and enhance the level of customer relationship management (CRM).
\end{abstract}

Keywords: Pareto/NBD Model; Customer Lifetime Value; Personalized Information; Customer Segmentation

\section{Introduction}

With the development and application of the computer and information technology, an increasing number of enterprises begin to store and study a great deal of customers' data. The methods and techniques which measure the Customer Lifetime Value (CLV) are gradually used to analysis and predict customers' future purchasing which provides much guidance for the marketing management.

Pareto/NBD model which is used to describe the customer repeat purchase behavior was firstly proposed by Schmittlein, Morrison and Colombo in 1987. Based on the assumption that the customer purchase behavior conform to certain rules or distribution, Pareto/NBD model may give a reasonable prediction of customers' future purchase frequency by using the historical purchase data. Because of the consideration to the possibility of customer churn and the growth of future buying while predicting customers' future purchase behavior, Pareto/NBD model has relatively high prediction accuracy.

Pareto/NBD model has been used by scholars from different industry who verified the applicability and validity of the model and achieved rich results. But scholars gradually found some disadvantages of the model in the process of research. When studying and analyzing, we found that due to the excessive reference of the general and little consideration of personalized information of customer, the predicted results of the model became average which results in a large deviation between the model prediction results and the actual buying behavior in the future. Therefore, this article puts forward an improved Pareto/NBD model that based on the personalized information of customer. 


\section{Literature Review}

At the beginning of the customer lifetime value research, scholars have put forward some prediction model for describing the customer repeat purchase behavior. Three models have a great influence, i.e. NBD (Negative Binomial Distribution) model, LSD (Logarithmic Series Distribution) model, NBD/Dirichlet model. Although these models can describe the customer purchasing behavior partly, but compared with the actual purchase there is a very big disparity.

In 1987, Schmittlein and Morrison proposed Pareto/NBD model used for predicting customers' future purchase frequency, for the firstly time, considering the phenomenon of customer churn. In 1994, Schmittlein and Peterson put a prediction model of customer purchase amount into the Pareto/NBD model and make an empirical analysis of distributive trades in industrial products market. In 2003, Reinartz and Kumar made directly use of Pareto/NBD model to estimate the lengths of time of customer relationship in the study of the relation between customer relationship and customer profitability, which had achieved good effects.

In 2005, the concept of value curve was introduced into the Pareto/NBD model. Combined with RFM (Recency, Frequency, Purchase amount) model and Pareto/NBD model, Fader and Hardie expanded the Pareto by the data of CDNOW online sellers and put forward a new customer lifetime value measurement model. In addition, they firstly realized the combination of RFM model with Pareto/NBD model, and achieved very good results.

For the firstly time, the Fader and Hardie made an improve of Pareto/NBD model itself in 2005. They made use of BG/NBD model instead of Pareto/NBD model to predict future customer purchasing behavior, and compared the predictions of these two models through the data of CDNOW online sellers. Finally, although these two models showed similar results, but the BG/NBD model greatly simplified the parameter estimation process of the Pareto/NBD model. In 2007, Glady and other scholars made an improve of the prediction accuracy of the Customer Lifetime Value (CLV) by relaxing the independence assumption of the purchase frequency model and purchase purchase amount model. In 2011, based on the simulation of customer churn process in Pareto/NBD model, Jerath, Fader and Hardie put forward the PDO (Periodic Death Opportunity) model, which greatly improved the prediction accuracy of the customer purchasing behavior.

Based on the data from the Beijing branch of Digital China company, Qi Jiayin and Li Huaizu made an empirical analysis of Pareto/NBD model and verified the validity of the model in 2003.

Based on the 5 years' sales data from a video equipment retailers, Ma Shaohui and Liu Jinlan made an empirical analysis and proved the prediction effect of Pareto/NBD model in 2006. Meanwhile, combining the Pareto/NBD model and purchase amount model, they have calculated the customer lifetime value which was used for early warning of the customer lifetime value. Finally, the predicting level of customer churn was also improved.

In 2007, Li Deqiang and Fu Qiansheng used Pareto/NBD model to calculate the customer potential value through the historical transaction database of some retail enterprise. Combined with clustering analysis of the current customer value, they got a subdivided customer base and its characteristics, which were used as the basis for enterprises to carry out the marketing work.

Ma Shaohui got the posterior distribution sample of Pareto/NBD model by introducing the markov chain monte carlo simulation (MCMC) method in 2008. Based on the result, he computed customer activity and predicted future transaction frequency, so as to avoid the bayesian integral problem of the original model and loosen the hypothesis of Pareto/NBD model. 
In 2011, on the basis of the subject-object value relationship analysis model, for the firstly time, network information was used by Ma Feicheng and Su Xiaomin as the research object. They studied the failure of network information through Pareto/NBD model and made an empirical analysis of the data of People's Daily online powerhouses BBS. Finally, they verified the feasibility and validation of the Pareto/NBD model in analyzing the failure discrimination of network information.

Through above researches we can find that the Pareto/NBD model has no doubt to predict future customer purchasing behavior, for the deficiency of this model, scholars have put forward the improvement method and obtained certain achievements. In this paper, aiming at the shortcomings of the Pareto/NBD model, the personalized information of customer has been used to improve the prediction accuracy of the Pareto/NBD model.

\section{Model Construction}

\subsection{Introduction of Pareto/NBD Model}

While the Pareto/NBD model is applied to predict future customer purchase frequency, customer purchasing behavior must comply with certain strict assumptions: firstly of all, because the customer churn is a random event and may occur at any time, therefore the enterprise is unable to observe the time when customer loss. What we can observe is only the fact that the customer doesn't have purchasing behavior in a long time. Secondly, for the time interval of customer purchases is random in the active period, so customer purchases may occur at any time, that is to say the last customer purchase does not affect the next. Finally, the rate of churn and purchase from different customers have great heterogeneity.

We assume that each purchase amount is $\Gamma(\delta, \eta)$ distributed, the different parameter of purchase amount between customers $(\eta)$ is $\Gamma(\mathrm{u}, v)$ distributed, namely, each purchase amount of customers in the future (m) is Gamma - Gamma mixed distributed:

The application of the Pareto/NBD model is based on the following five basic assumptions:

1. Repeat purchase behavior of individual customers is Poisson distribution whose rate of purchase is $\lambda$. If customer is in active period, the probability of $\mathrm{x}$ times purchase behavior within time $t$ is as follow:

$$
P[X=x \mid \lambda(t), t]=e^{-(\lambda t)^{x}} \bullet \frac{(\lambda t)^{x}}{x !}, x=0,1,2,3 \ldots
$$

2. The rate of purchase $\lambda$ is Gamma $\Gamma(r, \alpha)$ distributed, namely:

$$
f(\lambda \mid r, \alpha)=\frac{\alpha^{r}}{\Gamma(r)} \lambda^{r-1} e^{-\alpha \lambda}, \lambda>0, r>0, \alpha>0
$$

Therefore, the average rate of purchase from different customers is $E[\lambda]=r / \alpha$ and the variance is $r / \alpha^{2}$, parameter $r$ reflects the homogeneous of transaction rate between different customers:

3. The time from firstly purchase to leave the company of individual customers obeys index distribution whose churn rate is $\mu$, the density function of which is:

$$
f(t \mid \mu)=\mu e^{-\mu t}
$$

4. The churn rate $\mu$ from different customers is Gamma $\Gamma(s, \beta)$ distributed:

$$
g(\mu \mid s, \beta)=\frac{\beta^{s}}{\Gamma(s)} \mu^{s-1} e^{-\beta \mu}, s, \beta>0
$$


Therefore, the churn rate from different customers is $E[\mu]=s / \beta$ and the variance is $s / \beta^{2}$, parameter s reflects the homogeneous of churn rate between different customers:

5. Customer's purchase rate $\lambda$ and customer's churn rate ${ }^{\mu}$ are independent of one another.

Then, main results of Pareto/NBD model could be deduced as following:

1. According to the foregoing assumptions, for a customer whose information is $\left(\mathrm{x},{ }^{t_{x}}, \mathrm{~T}\right)$, namely, he has $\mathrm{x}$ times repeat purchase during $(0, T)$ and his final transaction happens at $t_{x}\left(t_{x}<T\right)$. Thus, the probability of no churn at $\mathrm{T}$ time is as follow:

$$
\begin{aligned}
& P\left(\text { active } \mid r, \alpha, s, \beta, X=x, t_{x}, T\right) \\
& =\int_{0}^{\infty} \int_{0}^{\infty} P\left(\text { active } \mid \lambda, \mu, X=x, t_{x}, T\right) f\left(\lambda, \mu \mid r, \alpha, s, \beta, X=x, t_{x}, T\right) d \lambda d \mu \\
& =\left\{1+\left(\frac{s}{r+s+x}\right)(\alpha+T)^{r+x}(\beta+T)^{s} A_{0}\right\}^{-1}, \alpha \neq \beta
\end{aligned}
$$

$$
\begin{aligned}
& \text { If } \quad \alpha>\beta, \\
& A_{0}=\frac{{ }_{2} F_{1}\left(r+s+x, s+1 ; r+s+x+1 ; \frac{\alpha-\beta}{\alpha+t_{x}}\right)}{\left(\alpha+t_{x}\right)^{r+s+x}}-\frac{{ }_{2} F_{1}\left(r+s+x, s+1 ; r+s+x+1 ; \frac{\alpha-\beta}{\alpha+T}\right)}{(\alpha+T)^{r+s+x}}
\end{aligned}
$$$$
\text { If } \alpha<\beta \text {, }
$$$$
A_{0}=\frac{{ }_{2} F_{1}\left(r+s+x, s+1 ; r+s+x+1 ; \frac{\beta-\alpha}{\beta+t_{x}}\right)}{\left(\beta+t_{x}\right)^{r+s+x}}-\frac{{ }_{2} F_{1}\left(r+s+x, s+1 ; r+s+x+1 ; \frac{\beta-\alpha}{\beta+T}\right)}{(\beta+T)^{r+s+x}}
$$

If $\alpha=\beta$,

$P\left(\right.$ active $\left.\mid r, \alpha, s, \beta, X=x, t_{x}, T\right)=\left\{1+\left(\frac{s}{r+s+x}\right)\left[\left(\frac{\alpha+T}{\alpha+t_{x}}\right)^{r+x+s}-1\right]\right\}^{-1}$

Where $\mathrm{T}$ is the length of historical transaction time, $t_{x}$ is the time when the last purchase behavior happens, $x$ is the repeat purchase frequency during time T. ${ }_{2} F_{1}(\bullet)$ is Gauss Hypergeometric Function. The formulas showed above have been simplified compared with primary formula.

2. For any randomly selected customer, the expected value of repeat purchase frequency during $(0, T)$ is as follow:

$$
E[X(t) \mid r, \alpha, s, \beta]=\frac{r \beta}{\alpha(s-1)}\left[1-\left(\frac{\beta}{\beta+t}\right)^{s-1}\right]
$$

3. For the customer who has $x$ times repeat purchase during $(0, T)$ and whose final transaction happens at $t_{x}\left(t_{x}<T\right)$, his expected value of purchase frequency during $(T, T+t)$ is as follow: 


$$
E\left[\mathrm{Y}(\mathrm{t}) \mid \mathrm{X}=\mathrm{x}, \mathrm{t}_{\mathrm{x}}, \mathrm{T}, r, \alpha, s, \beta\right]=\frac{(\mathrm{r}+\mathrm{x})(\beta+T)}{(\alpha+T)(s-1)}\left[1-\left(\frac{\beta+T}{\beta+T-t}\right)^{s-1}\right] \bullet P\left(\operatorname{active} \mid r, \alpha, s, \beta, X=x, t_{x}, T\right)
$$

\subsection{Disadvantages Analysis of Pareto/NBD Model}

Customer churn and customer purchases are carefully considered in Pareto/NBD model while predicting customer purchase frequency in the future. Pareto/NBD model win scholars' consistent high praise for its relative high accuracy. However, through the empirical analysis we find that Pareto/NBD model has a large deviation while predicting future and actual purchase frequency of individual customers.

As shown in the Figure 1, we randomly selected 30 customers whose previous average purchase is 3 and compared their future actual purchase frequency with the prediction results from Pareto/NBD model. At last, we found that Pareto/NBD model has some deviation although it has certain prediction ability.

This paper analyzes the difference between the predicted and the actual purchase frequency of each customer. We parse the derivation process of Pareto/NBD model once again and find the deep cause that result in deviation from the actual result in the application of Pareto/NBD model. That is we have too much reference on overall sample information and weaken the influence on the result of the late to purchase caused by individual customer's personalized information when using Pareto/NBD model to calculate customers' purchase frequency in the future.

From the formula (10) used to predict purchase frequency we can see that the influence of early purchase information for later prediction results is not fully embodied in the formula, thus the prediction results show a relatively flat distribution and the range of the prediction results is much smaller than the actual customer purchase frequency. So it is hard to ensure the accuracy. To sum up, the less application of personalized information eventually lead to the decline of the prediction accuracy of Pareto/NBD model.

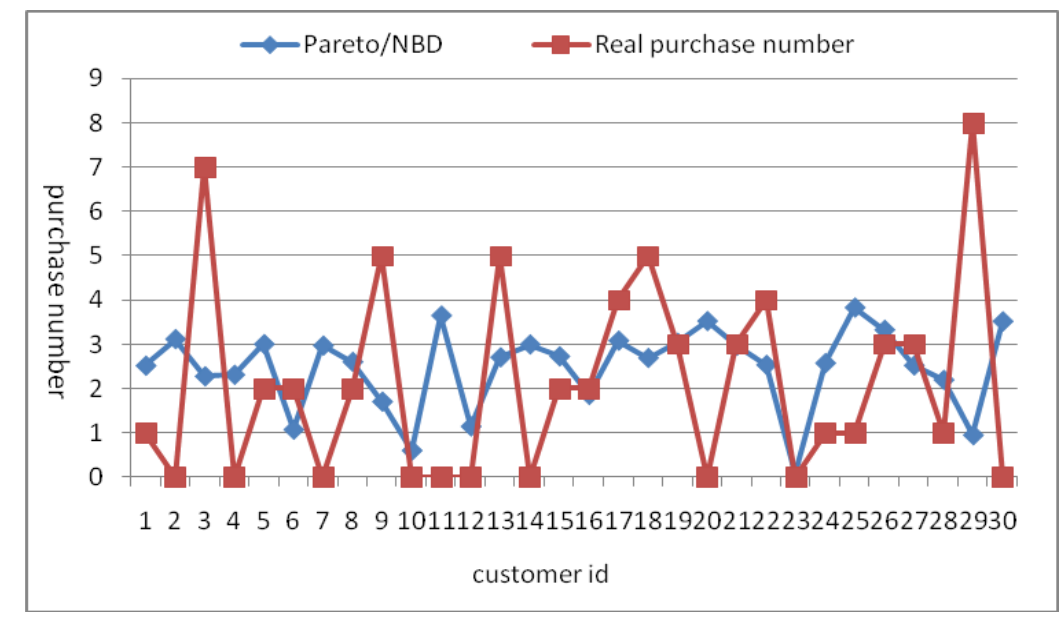

Figure 1. Comparative Analysis of the Real Purchase Number and the One Calculated by the CLV Model

\subsection{Improvement of Pareto/NBD Model}

\subsubsection{Improvement Analysis of Pareto/NBD Model}

Through above analysis we can see that the root cause of the bad prediction results is the little use of the personalized information of individual customer. Therefore, this paper puts forward the method which added the personalized information of individual customer to improve the Pareto/NBD model, namely the regulating effect of the personalized 
information is applied to improve the accuracy of the prediction of the future purchase frequency.

Personalized information that effectively describes the various properties of the individual can reflect each characteristic of human beings. This information can. From the perspective of marketing, the customer personalized information contain customer's demographic information such as ID, gender, age, occupation, etc. and consumer behavior data such as purchase frequency, purchase amount, purchase categories and psychological characteristics such as values, lifestyle, preferences and so on.

Because the consumer behavior information such as purchase frequency and purchase amount directly affect the future customer purchasing behavior and it is easy to do quantitative analysis, so this kind of personalized information is very suitable for us to combine with Pareto/NBD model to improve the influence on the predicting results of Pareto/NBD model.

On the contrary, it is difficult to quantize the demographic information and the psychological characteristics of the customer. The information showed above is not suitable for being used in the quantitative model. Therefore, the personalized information such as the customer purchasing behavior data is used as the adjustment factor to improve Pareto/NBD model.

On the basis of using RFM model to calculate RFM value of each customer, this paper realize the way of integrating personalized information to predict customer purchase frequency. There are some reasons why we choose the RFM model. On the one hand, as three elements of RFM model, customer purchase frequency and the recent purchase time and purchase amount are all the customers' personalized information. On the other hand, RFM value of each customer is a kind of adjustment factor contains specific personalized information and can be used to adjust the influence on the predicting results of Pareto/NBD model.

\subsubsection{Combination Personalized Information with Pareto/NBD Model}

This paper uses $e^{x}$ as adjustment function in the process of improvement, specific operation steps are as follows:

(1) Where $t_{x}, x, m$ represent the value of $\mathrm{R}, \mathrm{F}$ and $\mathrm{M}$. In order to eliminate the diversity caused by different dimension, firstly of all, the author standardizes the three indicators:

$$
\left\{\begin{array}{l}
x^{\prime}=(x-\bar{x}) /\left(x_{l}-x_{s}\right), \\
t_{x}{ }^{\prime}=\left(t_{x}-\overline{t_{x}}\right)\left(t_{x l}-t_{x s}\right), \\
m^{\prime}=(m-\bar{m})\left(m_{l}-m_{s}\right) .
\end{array}\right.
$$

Where $t_{x}{ }^{\prime}, x^{\prime}, m^{\prime}$ represent standardized $\mathrm{R}, \mathrm{F}$ and $\mathrm{M}$.

(2) From standardized R, F and M above, we can calculate each customer's RFM value, formula is as follow:

$$
\varpi^{\prime}=\left(x^{\prime}+t_{x}{ }^{\prime}+m^{\prime}\right) / 3
$$

(3) Add each customer's RFM value $\varpi^{\prime}$ into Pareto/NBD model. For the customer who has $\mathrm{x}$ times repeat purchase during $(0, T)$ and whose final transaction happens at $t_{x}\left(t_{x}<T\right)$, the predictive value of purchase frequency $E\left[\mathrm{Y}^{*}(\mathrm{t})\right]$ occurred during $(T, T+t)$ is calculated as follow: 


$$
\begin{aligned}
& E\left[\mathrm{Y}^{*}(\mathrm{t})\right]=E\left[\mathrm{Y}(\mathrm{t}) \mid \mathrm{X}=\mathrm{x}, \mathrm{t}_{\mathrm{x}}, \mathrm{T}, r, \alpha, \mathrm{s}, \beta\right] \times \mathrm{e}^{(\mathrm{RFM} \text { 值 })} \\
& =\frac{(\mathrm{r}+\mathrm{x})(\beta+T)}{(\alpha+T)(s-1)}\left[1-\left(\frac{\beta+T}{\beta+T-t}\right)^{s-1}\right] P\left(\text { active } \mid r, \alpha, s, \beta, X=x, t_{x}, T\right) \llbracket \mathrm{e}^{\sigma^{\prime}} \\
& =\frac{(\mathrm{r}+\mathrm{x})(\beta+T)}{(\alpha+T)(s-1)}\left[1-\left(\frac{\beta+T}{\beta+T-t}\right)^{s-1}\right] P(\text { active }) \llbracket \mathrm{e}^{\left(x^{\prime}+t_{x}^{\prime}+m\right) / 3}
\end{aligned}
$$

\section{Empirical Analysis}

\subsection{Data Description}

The time in this paper is measured in weeks. In this paper, we use the actual sales data from a retail store in Dalian as the research sample and use the firstly purchase data occurred during the firstly 12 weeks in 2007 as empirical simple. All the simple data contains 78weeks transaction data from 2000 customers from January of 2007 to June of 2008. The firstly half of the data is used to estimate parameter of Pareto/NBD model and the second half of the data is used to analyze and compare the predicting ability between improved model and Pareto/NBD model.

Figure 2 shows the simulation of customer repeat purchase behavior. The firstly day of 2007(January 1, 2007) is the zero time. The time $t_{0}$ is the firstly purchase time. The time $\mathrm{T}$ is the time of 39th week of 2007 minus the time $t_{0}$ represents the firstly purchase time. The time $t_{x}$ is the last purchase time minus the firstly purchase time, so $t_{x<=\mathrm{T}}$.

For example, for the firstly purchase occurred at January $29, \mathrm{~T}=35$; for the last purchase occurred at September 21, x represents purchase frequency, $t_{x}=31$.

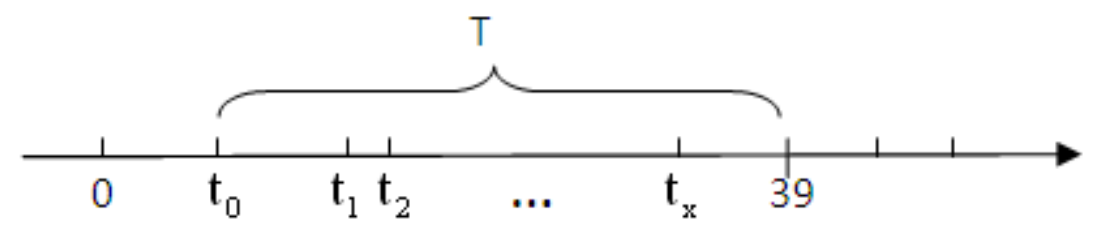

Figure 2. Mimic Diagram of Customer Purchase Behavior

\subsection{Parameter Estimation}

We use maximum likelihood estimation method to estimate parameter in this paper and use MATLAB to realize parameter estimation. The logarithmic likelihood function of Pareto/NBD model is as follow:

$$
L L(r, \alpha, s, \beta)=\prod_{i=1}^{N} L\left(r, \alpha, s, \beta \mid X=x_{i}, t_{x_{i}}, T_{i}\right)
$$

While,

$L\left(r, \alpha, s, \beta \mid X=x_{i}, t_{x_{i}}, T_{i}\right)=\left[\frac{1}{\left(\alpha+T_{i}\right)^{r+x_{i}}\left(\beta+T_{i}\right)^{s}}+\left(\frac{s}{r+s+x_{i}}\right) A_{0}\right] \bullet \frac{\Gamma\left(r+x_{i}\right) \alpha_{r} \beta^{s}}{\Gamma(r)}$

Results: $\gamma=1.0444, \alpha=6.5976, s=0.5611, \beta=32.7867, \mathrm{LL}=24200$.

From the results showed above we can see that the average rate of purchase is $0.1589(\gamma / \alpha=0.1589)$ when the customers are in active period. Namely, 8 times each year and the maintenance time between customer and company is $58(\beta / \mathrm{s}=58)$. 


\subsection{Analysis of Customer Purchase Frequency}

According to the purchase data $\left(x, t_{x}, T\right)$ from the firstly half , we use formula (10) and (13) to calculate two types of purchase frequency of each customer during the predictive period. According to the purchase frequency, all the customers are divided into 8 groups $(0,1,2,3,4,5-6,7-10,11+)$. We use improved model and Pareto /NBD model to predict the estimated value and actual value of average purchase frequency during 39 weeks in the future. As showed in figure 3, although the predictive results of two models are similar, but we also can find the fact that the predictive value from improved value is more close to the actual value.

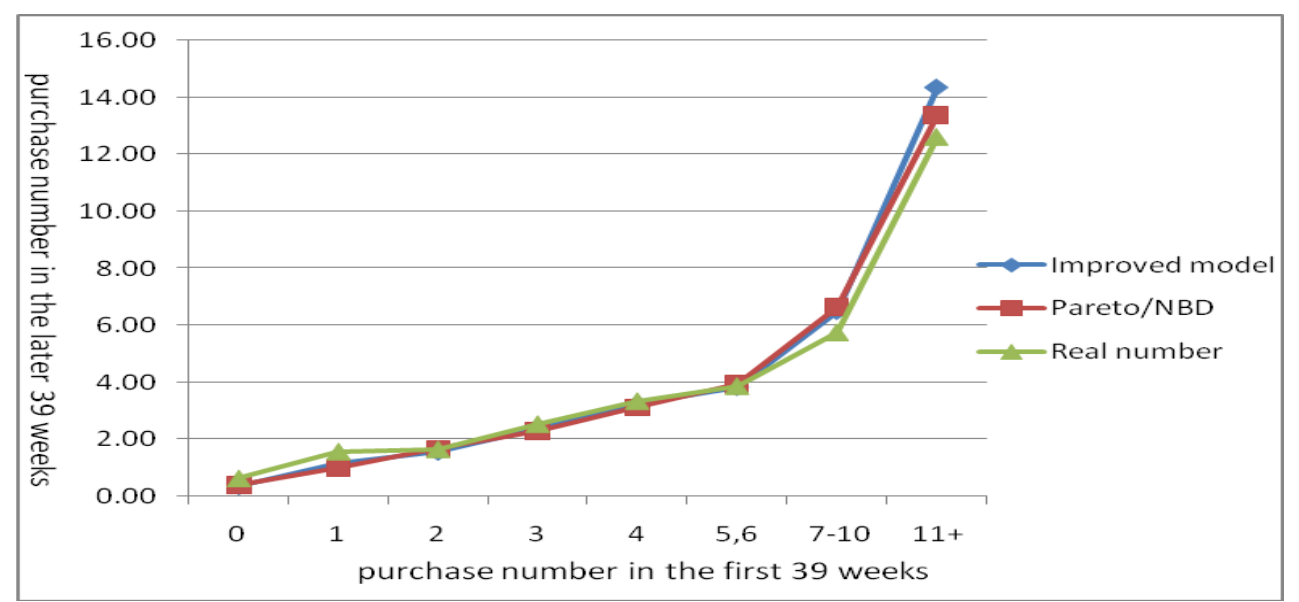

Figure 3. Conditional Expectations of Purchase Number

This paper analyzes the absolute error between predictive purchase frequency from each model and actual customer purchase frequency. As we can see in Table 1 that the average absolute error of improved model is smaller than that of Pareto/NBD model, which prove the feasibility and validity of the improved model considering customers' personalized information.

Table 1. Comparative Analysis of the Residual of Purchase Number

\begin{tabular}{lll}
\hline & Pareto/NBD model & Improved model \\
\hline average absolute error & 2.322 & 2.183 \\
\hline
\end{tabular}

\section{Application of the Improved Model}

\subsection{Calculation of Customer Lifetime Value}

Customer Lifetime Value (CLV) is a discounted value of cash flow produced from the transaction between customers and enterprises in the Customer life cycle, the calculation formula can be represented as:

$$
C L V=\sum_{t=1}^{n} \frac{f_{t} \bullet m_{t}}{(1+d)^{t}}
$$

Where $f_{t}$ is the expected purchase frequency in stage $t, m_{t}$ is the single purchase amount in the future, $\mathrm{d}$ is the discounted value, $n$ is the total length of time of customer relationship. 
This paper uses the improved Pareto /NBD model to estimate the customer purchase frequency in the future. For the customer whose historical purchase information is $\left(X=x, t_{x}, T\right)$, the purchase frequency in the stage $\mathrm{t}$ in the future can be represented as:

$$
f_{t}=E\left[\mathrm{Y}(\mathrm{t}) \mid \mathrm{X}=\mathrm{x}, \mathrm{t}_{\mathrm{x}}, \mathrm{T}\right]-E\left[\mathrm{Y}(\mathrm{t}-1) \mid \mathrm{X}=\mathrm{x}, \mathrm{t}_{\mathrm{x}}, \mathrm{T}\right]
$$

This paper uses the Gamma-Gamma model to estimate the customer purchase amount in the future. We assume that each purchase amount is $\Gamma(\delta, \eta)$ distributed, the different parameter of purchase amount between customers $(\eta)$ is $\Gamma(\mathrm{u}, v)$ distributed, namely, each purchase amount of customers in the future (m) is Gamma - Gamma mixed distributed:

$$
\begin{aligned}
& \mathrm{GG}(\mathrm{m} \mid \delta, \mathrm{u}, v)=\int_{0}^{\infty} \frac{\eta^{\delta}}{\Gamma(\delta)} m^{\delta-1} e^{-\eta m} \frac{v^{u}}{\Gamma(u)} \eta^{u-1} e^{-v \eta} d_{\eta} \\
& =\frac{\Gamma(\delta+u)}{\Gamma(\delta) \Gamma(u)}\left(\frac{m}{v+m}\right)^{\delta}\left(\frac{v}{v+m}\right)^{u} \frac{1}{m}
\end{aligned}
$$

For the customer whose historical purchase information is $(x, \bar{m})$, the calculation formula of each purchase amount in the future can be represented as:

$$
E(m \mid \delta, u, v, \bar{m}, x)=\frac{\delta(x \bar{m}+v)}{\delta x+u-1}=\frac{u-1}{\delta x+u-1} \bullet \frac{v \delta}{u-1}+\frac{\delta x}{\delta x+u-1} \bullet \bar{m}
$$

Therefore, the average purchase amount in stage $t$ is

$$
m_{t}=E(m \mid \delta, u, v, \bar{m}, x)
$$

For the sample of 2000 customers above, estimation of customers' CLV can be done according to the formula (15)-(18). When the length of time of customer relationship (n) is 39 weeks and the annual discount rate (d) is $15 \%$, we can calculate each customer's CLV in 39 weeks in the future: the minimum value (CLV) is 13 Yuan, the mid-value (CLV) is 1609 Yuan, the maximum value (CLV) is 387560 Yuan, the average (CLV) is 5094 Yuan. These CLV values show the value of the customer may bring for the enterprise, which can be used as the foundation of marketing work.

\subsection{The Customer Segmentation and Marketing Strategy Based on CLV}

From the statistics of the CLV result shows above, we can see that there is a great difference between different customer purchases. If the enterprise take same measures and spend same marketing resources for all customers, this may result in a lower degree of care for the high value customers. Meanwhile too much input for the low value customers may result in enormous waste of enterprise resources. For this background, a classification based on CLV result is presented and also the differentiated marketing ideas.

According to the law of "20/80/30", 80\% profits of the company comes from the High-quality customers that occupy $20 \%$ of all customers and the final $30 \%$ customers have a negative influence on the enterprise value. So, enterprises should try to reduce the input. This article defines the maximum of $20 \%$ customers that have a larger CLV result as High- quality clients and the minimum of $30 \%$ customers that have a smaller CLV result as loose customers.

In addition, in order to facilitate differentiated marketing and personalized marketing, this article chooses the $4 \%$ customers who have the highest CLV result as Core 
customers. As the most valuable resource, the enterprise must put the most marketing effort in the elite customer. Finally, the article defines the 50\% customers who have the middle CLV result as the developing customers that have their certain value to the enterprise.

As show in Figure 4, the inner ring represents the percent of customers and the outer ring represents the percent of purchase amount. The gray line area represents the Core customers. The pure white area represents the High-quality customers. The pure gray area represents the Developing customers. The white line area represents the loose customers. As shown in the Figure 4, the $4 \%$ Core customers and the $16 \%$ High-quality customers contribute the $59 \%$ purchases. While the $50 \%$ developing customers contribute the $34 \%$ purchases and the $30 \%$ loose customers contribute only $7 \%$ purchases. Thus it can be seen that on the basis of the model in this paper, the new customer classification method can distinguish different customers effectively, which lay a foundation for the enterprise to implement differentiated marketing.

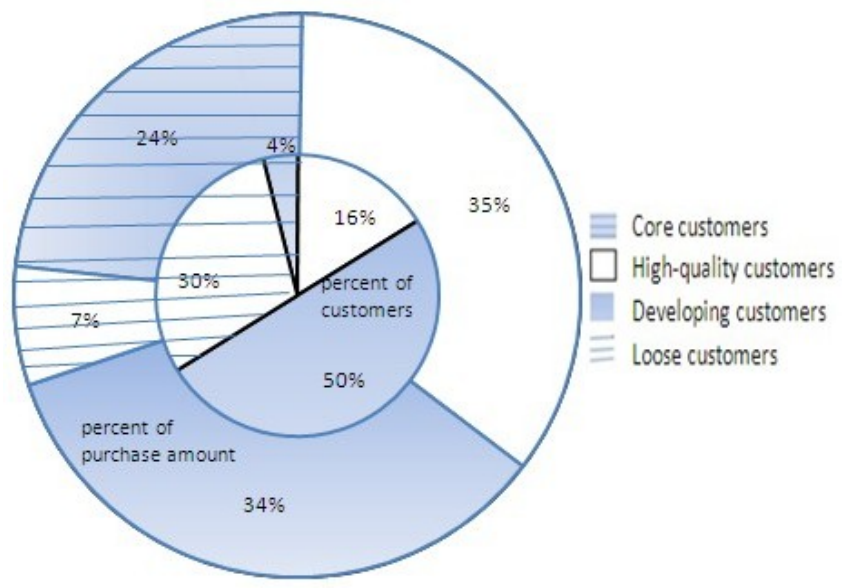

Figure 4. Comparative Analysis of Numbers and Purchases of Different Customer Bases

On the basis of the application of CLV customer segmentation, this paper puts forward different marketing strategies for each customer group.

(1) The main source of the corporate profits comes from the Core customers who have strong consumption ability and have the high perception to the enterprise products and services. The Core customers are the loyal customers who deserve our maintenance. For the Core customers, enterprises can set up a dedicated team to keep close contact with them and meet their needs as soon as possible. The enterprise should let them feel the special care and the most comprehensive and most considerate service, which may avoid the negative influence on enterprises caused by the Core customer churn.

(2) The High-quality customers also are the main profits source of enterprises. Enterprises have to maintain the relationship with them and take the appropriate marketing measures to motivate them to increase the purchase frequency or amount, so that they can develop the Developing customers into the Core customers. In addition, because the Developing customers still have certain gap compared with the Core customers, so they are likely to be the competitors' High-quality customer. Enterprises should pay close attention to their buying behavior and do a good early warning to prevent them from losing.

(3) Although the developing customers' purchase ability is limited and the most purchased goods are located at low price, but they have a big base number and vast internal diversity. So enterprises should pay more attention to the potential High-quality customers. For example, those young customers who don't have strong consumption 
ability are our potential customer. Enterprises should realize that these customers may grow up to be more advantageous to them with the ascension of the job status and economic strength. In order to be ready for the long-term development, the enterprise should pay attention to put resources into them.

(4) Because the loose customers don't have regular pattern, so the arrival or churn of them have no influence on enterprises sales and profits. Enterprises should reduce the marketing investment and put more resources into serving the loyal High-quality customers.

In addition, there are a lot of differences between internal customer groups. For some customers who have reach the average level of High-quality customers, although they don't have enough purchase amount and are of little value to the enterprise profits, but they have great value of word of mouth. Enterprises should strive to maintain these customers. Therefore, on the condition of enough resource, enterprises should do further study of various customer groups to find more valuable information and get more valuable customers to make the enterprise profits continued to grow.

\section{Conclusions}

This paper gives a summary of relative research achievements of the Pareto/NBD model. To the question of Pareto/NBD model when predicting customers' future purchase frequency, we put forward an improved Pareto/NBD Model that based on the personalized information of customer to improve the prediction accuracy. Based on the sample of the actual sales data of a retail market in Dalian, we compared the capability in predicting customers' future purchase frequency of the Pareto/NBD model and the improved Pareto/NBD model and verified the validity and superiority of the improved Pareto/NBD model. In addition, this paper uses the improved Pareto/NBD model to forecast customers' future purchase frequency and the Gamma - Gamma model to predict customers' future per purchase amount and finally calculates each customer's Customer Lifetime Value (CLV) and puts forward the segmentation method based on CLV and the corresponding management strategy, which may provide guidance for enterprises to implement the differentiation marketing strategy and enhance the level of Customer Relationship Management (CRM).

\section{Acknowledgements}

Finally, with the help of project supported by Ministry of Education Humanities and Social Science Fund Project Funding Projects (10YJC630065) and Fundamental Research Funds for the Central Universities (DUT12RW408), we finished this paper successfully. So we appreciate greatly that the above projects give us lots of funding.

\section{References}

[1] D. C. Schmittlein, D. G. Morrison and R. Colombo, "Counting Your Customers: Who Are They and What Will They Do Next?”, Management Science, vol. 33, (1987), pp. 1- 24.

[2] A. S. C. Ehrenberg, "Repeat Buying”, Oxford University Press, USA, (1988).

[3] D. C. Schmittlein and R. A. Peterson, "Customer Base Analysis: An Industrial Purchase Process Application”, Marketing Science, vol. 13, (1994), pp. 41- 67.

[4] W. Reinartz and V. Kumar, "On the Profitability of Long Life Customers in a Noncontractual Setting: An Empirical Investigation and Implications for Marketing”, Journal of Marketing, (2000), vol. 64, pp. 17-35.

[5] W. Reinartz and V. Kumar, "The Impact of Customer Relationship Characteristics on Profitable Lifetime Duration”, Journal of Marketing, vol. 67, (2003), pp. 77- 99.

[6] P. S. Fader, B. Hardie, K. L. R. F. M. Lee and C. L. V., "Using Iso-Value Curves for Customer Base Analysis”, Journal of Marketing Research, vol. 42, (2005), pp. 135-144.

[7] P. S. Fader, B. Hardie and K. L. Lee, “Counting Your Customers”, Easy Way: An Alternative to the Pareto/NBD Model, Marketing Science, vol. 24, (2005), pp. 275- 284. 
[8] N. Glady, B. Baesens and C. Croux, "A modified Pareto/NBD Approach for Predicting Customer Lifetime Value”, Expert Systems with Applications, forthcoming, vol. 36, (2009),pp. 21-42.

[9] K. Jerath, P. S. Fader and B. G. S. Hardie, "New Perspectives on Customer "Death" Using a Generalization of the Pareto/NBD Model”, Marketing Science, vol. 30, (2011), pp. 866-880.

[10] J. Qi, H. Li and H. Shu, "SMC Model in IT distribution industry: An Case Empirical Research", Systems Engineering-Theory \& Practice, vol. 24, (2004), pp. 70-78.

[11] S. Ma and J. Liu, "An Empirical and Applied Research on the Pareto/NBD Model”, Journal of Management Science, vol. 19, (2006), pp. 45-49.

[12] D. Li and Q. Fu, "SMC Model in the Discovery of Potential Customer Value: An Applied and Empirical Research”, Value Engineering, vol. 21, (2007), pp. 29- 55.

[13] S. Ma, “The Pareto/NBD Model Extension”, Systems Engineering, vol. 26, (2008), pp. 123- 127.

[14] F. Ma, X. Su and J. Wang, "An Exploratory Study of Pareto / NBD Model in the Discriminant Analysis of the Failure Network Information”, Information Studies: Theory \& Application, vol. 34, (2011), pp. 50- 55.

\section{Authors}

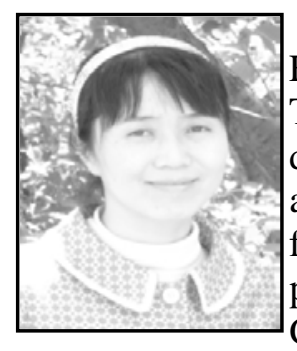

Yanhong Guo is currently an Assistant Professor in the Faculty of Management and Economics, Dalian University of Technology, China. She received the Ph.D. degree and the M.S. degree from Dalian University of Technology. Her research areas are marketing models and recommender systems. Her research is funded by Fund of Ministry of Education of China. She has published in refereed conference proceedings and journals in China.

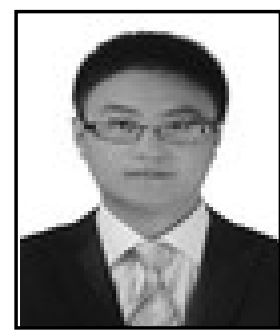

Hui Wang is currently a postgraduate student and pursuing for master degree in the Faculty of Management and Economics, Dalian University of Technology, China. His study direction is marketing of business administration. He has published several papers in Chinese journals.

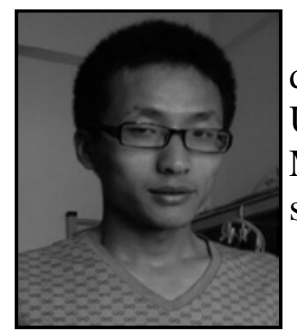

BoYin is a postgraduate student and pursuing for master degree in the Faculty of Management and Economics, Dalian University of Technology, China. He received her Bachelor of Management degree from Dalian University of Technology. His study direction is marketing of business administration.

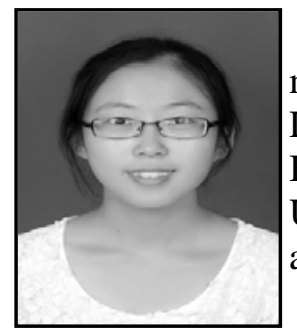

Wei Liu is currently a postgraduate student and pursuing for master degree in the Faculty of Management and Economics, Dalian University of Technology, China. She received her Bachelor of Management degree from China Agricultural University. Her study direction is marketing of business administration. She has published two papers in Chinese journals. 